\title{
Respiratory complications following mini-invasive laparoscopic and thoracoscopic esophagectomy for esophageal cancer. Experience in 215 patients
}

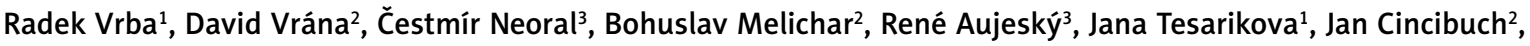 \\ Jana Zapletalová4, Tomáš Jínek ${ }^{5}$, Martin Stašek ${ }^{3}$ \\ ${ }^{1}$ Department of Surgery I, University Hospital, Olomouc, Czech Republic \\ ${ }^{2}$ Department of Oncology, University Hospital, Olomouc, Czech Republic \\ ${ }^{3}$ Department of Surgery I, Faculty of Medicine and Dentistry, Palacký University, Olomouc, Czech Republic \\ ${ }^{4}$ Department of Biophysics, Faculty of Medicine and Dentistry, Palacký University, Olomouc, Czech Republic \\ ${ }^{5}$ Department of Surgery, Nový Jičín, Czech Republic
}

Videosurgery Miniinv 2019; 14 (1): 52-59

DOI: https://doi.org/10.5114/wiitm.2018.77276

\begin{abstract}
Introduction: Respiratory complications $(R C)$ including respiratory failure and adult respiratory distress syndrome (ARDS) affect the outcomes of esophagectomy substantially. In order to decrease their incidence, identification of important features of $R C$ is necessary.

Aim: To evaluate the incidence and risk factors of postoperative $R C$ following hybrid esophagectomy.

Material and methods: The retrospective analysis of consecutive hybrid esophagectomies for malignancies (transhiatal laparoscopic or thoracoscopic resection and limited open reconstruction phase) assessed the incidence and outcomes of RC in relation to the patients' age, ASA score, neoadjuvant therapy, type of surgical procedure, TNM stage, the incidence of anastomotic leak and Clavien-Dindo classification.

Results: Transhiatal laparoscopic $(176,81.9 \%)$ or thoracoscopic hybrid esophagectomy (39, 18.1\%, conversion in 7 patients) was completed in 215 patients, 187 (87\%) men and 28 (13\%) women. Respiratory complications developed in 86 (40\%) and severe respiratory failure or ARDS occurred in 29 (13.5\%) patients. The overall in-hospital mortality was 7.4\%, 30-day mortality 5.6\% (RC 9, myocardial infarction 1, conduit necrosis 1), and 90-day mortality a further $1.8 \%$ (multiple organ failure, ARDS). The incidence of RC correlates significantly with ASA score II and III $(p=0.0002)$ and Clavien-Dindo grade 4 and 5 in severe RC $(p<0.0001)$. Furthermore, hospital stay $(p<0.0001)$ and mortality $(p<0.0001)$ were significantly increased in $R C$.

Conclusions: The results show a higher occurrence of $R C$ in polymorbid patients and patients with severe complications according to the Clavien-Dindo classification. Adequate risk management including surgical technique and perioperative prophylaxis and therapy of $R C$ should be studied and standardized.
\end{abstract}

Key words: neoadjuvant therapy, esophagectomy complication, respiratory complication, esophageal cancer therapy.

\section{Introduction}

The only potentially curative therapy for esophageal cancer is the esophagectomy in T1b and higher stages and endoscopic resection in the T1a stage. The treatment protocol includes neoadjuvant or adjuvant therapy consisting of systemic chemotherapy 
and external beam radiation in $\mathrm{T} 3$ and higher stages. The most serious risk of surgical treatment is presented by the development of respiratory complications (RC), with respiratory failure and adult respiratory distress syndrome (ARDS) as the leading causes of postoperative morbidity and mortality. Further important complications comprise ischemic conduit necrosis (gastric conduit in $0.5 \%$, colonic interposition in 13\%) [1] and anastomotic leakage (5-20\%) with a significant risk of severe mediastinitis development, especially in the thoracic anastomosis. The identification of risk factors for RC should lead to the implementation of tailored prophylactic and therapeutic intervention. The active approach may be the bridge to a further decrease in lethal and complicated postoperative outcomes.

\section{Aim}

The aim was to evaluate the incidence and outcomes of RC following hybrid esophagectomy for esophageal malignancies. Primary endpoints were the identification of basic risk factors for RC development and their relation to morbidity and mortality.

\section{Material and methods}

A retrospective single tertiary center analysis of the RC was applied to the cohort of esophageal cancer patients undergoing transhiatal or transthoracic esophagectomy in the period 2005-2016. The incidence of RC was assessed in relation to age
( $\leq$ versus $>60$ years), ASA score, neoadjuvant therapy, type of surgical procedure (transhiatal or transthoracic approach), the incidence of anastomotic leakage, TNM stage and Clavien-Dindo classification of postoperative complications (Table I) [2]. Esophageal cancer was diagnosed by endoscopy with biopsy, endosonography, and positron emission tomography (PET)/computed tomography (CT) examination. In the case of an upper third esophageal tumor, bronchoscopy was performed to exclude tumorous airway infiltration. General internal and nutritional status and spirometry were completed preoperatively with attributed ASA classification. Since 2012, the sputum and a throat swab for microbiological examination were collected from all patients 7 days prior to surgery. In the silent lower respiratory tract infection, targeted antibiotic therapy was administered and the procedure was postponed. In the case of minimal sputum contamination values without clinical signs of lower respiratory tract infection, antibiotic prophylaxis based on microbiological culture was administered.

Esophagectomy was primarily performed in all patients with stages T1,2 NO MO. Patients with higher $\mathrm{T}$ stages without evidence of metastatic disease were indicated for neoadjuvant therapy. This encompassed three cycles of chemotherapy (5-fluorouracil, cisplatin) and concomitant external beam radiotherapy (2 Gy per fraction to a total dose of $50 \mathrm{~Gy}$ ). In the case of stenosis with significant dysphagia, jejunostomy was carried out prior to the neoadjuvant

Table I. Clavien-Dindo classification of surgical complications

\begin{tabular}{|c|c|}
\hline Grade & Definition \\
\hline I & $\begin{array}{l}\text { Any deviation from the normal postoperative course without the need for pharmacological treatment or surgical, } \\
\text { endoscopic, or radiological interventions. Allowed therapeutic regimens include drugs such as antiemetics, anti- } \\
\text { pyretics, analgesics, diuretics, electrolytes, and physiotherapy. This grade also includes wound infections opened } \\
\text { at the bedside }\end{array}$ \\
\hline II & $\begin{array}{l}\text { Requirement for pharmacological treatment with drugs other than those allowed for grade I complications. Blood } \\
\text { transfusions and total parenteral nutrition are also included }\end{array}$ \\
\hline IIIa & Requirement for surgical, endoscopic or radiological intervention not under general anesthesia \\
\hline IIIb & $\begin{array}{l}\text { Requirement for surgical, endoscopic or radiological intervention under general } \\
\text { anesthesia }\end{array}$ \\
\hline IVa & $\begin{array}{l}\text { Life-threatening complications (including CNS complications) requiring IC/ICU management. Single organ } \\
\text { dysfunction (including dialysis) }\end{array}$ \\
\hline $\mathrm{IVb}$ & $\begin{array}{l}\text { Life-threatening complications (including CNS complications) requiring IC/ICU management. Multiple organ } \\
\text { dysfunction }\end{array}$ \\
\hline V & Death of the patient \\
\hline
\end{tabular}


treatment. Subsequent restaging (PET/CT and endoscopy) together with clinical and laboratory examination evaluated the effect of neoadjuvant treatment. The eligible patients were scheduled for esophagectomy within 8-12 weeks after neoadjuvant therapy completion. The hybrid esophagectomy technique consisted of a minimally invasive approach in the resection phase (transhiatal laparoscopic or thoracoscopic) and the limited open approach for reconstruction of the digestive tract. Transhiatal minimally invasive esophagectomy was indicated in a tumor location in the distal third of the esophagus. In thoracic esophageal tumors within $30 \mathrm{~cm}$ of the incisors, the transthoracic approach was selected. In the transhiatal approach, the standard mediastinal lymphadenectomy was performed up to the tracheal bifurcation. In the case of thoracoscopic access, an extended thoracic lymphadenectomy was performed. The reconstruction phase consisted of the limited open gastric conduit construction with extramucosal pyloromyotomy. The manual continuous single-layer anastomosis was constructed in the deep cervical space. The postoperative care required advanced intensive postoperative care. The nutrition was provided by a combination of enteral and parenteral therapy. On postoperative day 7 , a follow-up radiologic study with water-soluble contrast was performed in order to verify the reconstruction with subsequent gradual peroral food intake. The presence of RC was evaluated by the clinical examination, laboratory markers, chest radiographs, and CT findings.

The retrospective evaluation of a group of patients with esophageal cancer was approved by the local Ethical Committee.

\section{Statistical analysis}

IBM SPSS Statistics version 22 was used to analyze the data. The $\chi^{2}$ test or Fisher's exact test was used in the case of low frequencies to determine the association between the occurrence of RC and qualitative parameters. The association between the incidence of RC and the quantitative parameters was assessed using the Kruskal-Wallis test. The normality of the data was tested using the Shapiro-Wilk test. The level of statistical significance was set at 0.05.

\section{Results}

The surgery was provided in 215 patients, 187 (87\%) men and $28(13 \%)$ women with the age of 34-81 (median 61) years. Adenocarcinoma was found in $129(60 \%)$ patients, squamous cell carcinoma in 81 $(37.7 \%)$ and other tumor histology in 5 (2.3\%) cases. The neoadjuvant therapy was indicated in 174 $(80.9 \%)$ patients with the tumor stages of T 3-4, $\mathrm{N} 0-2, \mathrm{M} 0$. The stratification of the ASA classification consisted of ASA I in 7 (3.3\%), ASA II in 107 (49.3\%) and ASA III in 101 (47.4\%) cases. The transhiatal laparoscopic approach was used in $176(81.9 \%)$ and thoracoscopy in 39 (18.1\%) patients. In distal tumors, the resection phase was performed through the transhiatal laparoscopic approach. The tumors of the thoracic esophagus required conversion from thoracoscopic resection to thoracotomy in 7 (17.9\%) cases due to bulky masses with possible airway infiltration. The number of resected lymph nodes ranged from 2 to 38, with a median of 11 . The estimated blood loss ranged from 100 to $3500 \mathrm{ml}$ with a median of $400 \mathrm{ml}$ (Table II). Respiratory complications developed in $86(40 \%)$, and severe respiratory failure or ARDS occurred in 29 (13.5\%) patients. The multidisciplinary therapy (artificial pulmonary ventilation, tracheostomy, bronchoscopic therapy, antibiotics) was successful in 15 (51.7\%) patients. Less severe RC including pneumonia, pleural effusion, pneumothorax and pulmonary atelectasis occurred in 57 (26.5\%) patients. These complications were treated with targeted antibiotic therapy, chest drainage, and bronchoscopic flushing. Chylothorax was confirmed in $6(2.7 \%)$ patients, the ligation of the thoracic duct was performed in $2(0.9 \%)$ patients, while the conservative therapy was successful in other cases. Cardiac complications developed in a total of $21(9.7 \%)$ patients and led to one death due to myocardial infarction on postoperative day 4. Necrosis of the gastric conduit and subsequent septic shock resulted in one death on postoperative day 28. A cervical anastomotic leak was found in a total of $22(10.2 \%)$ patients. The condition required surgical revision with drainage in 7 (31.8\%) and implantation of a covered biodegradable stent in $2(9 \%)$ cases. Conservative management was applied in the other $13(59.2 \%)$ patients. A postoperative tracheoesophageal fistula was demonstrated in $2(9 \%)$ cases and required biodegradable esophageal stent implantation. The esophageal stenting was successful in all cases. Paralysis of the left recurrent nerve was confirmed in 19 (8.8\%) patients. The overall complication-related mortality reached $7.4 \%$ (16 patients). The 30-day mortal- 
Table II. Characteristics of the group of patients managed using minimally invasive hybrid laparoscopic and thoracoscopic esophagectomy

\begin{tabular}{|c|c|c|c|}
\hline Parameter & $N(\%)$ & MIE laparoscopic & MIE thoracoscopic \\
\hline \multicolumn{4}{|l|}{ Gender: } \\
\hline Male & $187(87)$ & 153 & 34 \\
\hline Female & $28(13)$ & 23 & 5 \\
\hline \multicolumn{4}{|l|}{ Age [years]: } \\
\hline$\leq 60$ & $92(42.7)$ & 72 & 20 \\
\hline$>60$ & $123(57.3)$ & 104 & 19 \\
\hline \multicolumn{4}{|l|}{ Tumor type: } \\
\hline Adenocarcinoma & $129(60)$ & 122 & 7 \\
\hline Squamous cell & $81(37.7)$ & 51 & 30 \\
\hline Other histology & $5(2.3)$ & 3 & 2 \\
\hline \multicolumn{4}{|c|}{ Neoadjuvant therapy: } \\
\hline Yes & $174(80.9)$ & 157 & 27 \\
\hline No & $31(19.1)$ & 19 & 12 \\
\hline \multicolumn{4}{|l|}{ ASA score } \\
\hline I & $7(3.3)$ & 6 & 1 \\
\hline$\|$ & $107(49.3)$ & 91 & 16 \\
\hline III & $101(47.4)$ & 79 & 22 \\
\hline
\end{tabular}

ity observed in the cohort was $5.6 \%$ (RC 9, myocardial infarction 1 , and necrosis of the conduit 1), and the further 90 -day mortality was $1.8 \%$ (multiple organ failure). The complications were assessed according to the Clavien-Dindo classification (Table II). A significant correlation of the incidence of $\mathrm{RC}$ with the ASA score $(p=0.0002)$ and the Clavien-Dindo classification ( $p<0.0001)$ was identified. In patients with RC, ASA classes II or III were found to be significantly more common. In patients with severe RC (respiratory failure or ARDS), Clavien-Dindo classes 4 and 5 were significantly more common as compared to less severe RC (pleural effusion, pneumothorax, pneumonia, atelectasis) or no complications (Table III). The incidence of RC significantly correlated with the length of hospital stay $(p<0.0001)$ and the postoperative fatal outcome $(p<0.0001)$ (Table IV). The implementation of active preoperative management of respiratory infections (sputum microbiology, antibiotic sensitivity, treatment of clinically silent infections, targeted prophylaxis) reduced the mortality by $2.4 \%$.

\section{Discussion}

Esophagectomy is one of the most demanding procedures in gastrointestinal surgery. The procedure can be performed using the open approach, the hybrid technique (combination of a classical and minimally invasive procedure) or the totally minimally invasive method [3]. Based on the current literary evidence, most centers prefer the minimally invasive approach in the resection phase combined with the limited open approach for gastrointestinal tract reconstruction $[4,5]$. The reported mortality of esophagectomy reaches $1-5.8 \%$, with the morbidity ranging from $17.9 \%$ to $58 \%[6,7]$ and the postoperative complication rate of $20-50 \%$ [8, 9]. Respiratory complications are the most common cause of death, ranging from 19.3\% (Mariette) to 44.4\% (Ott) [10-12]. Ott et al. reported $3.8 \%$ mortality in 240 patients following Ivor-Lewis esophagectomy for distal esophageal adenocarcinoma, but $50 \%$ of the deaths were caused by RC [6]. The overall mortality of the 379 cases reported by Atkins et al. was 5.8\% [7]. Pneumonia as the dominant respiratory cause of 
Table III. Statistical evaluation of respiratory complications (mild - pneumothorax, pleural effusion, pneumonia, atelectasis; severe - ARDS, respiratory failure) based on the considered parameters

\begin{tabular}{|c|c|c|c|c|}
\hline Parameter & $\begin{array}{l}\text { Without respiratory } \\
\text { complications }\end{array}$ & $\begin{array}{l}\text { Mild respiratory } \\
\text { complications }\end{array}$ & $\begin{array}{l}\text { Severe respiratory } \\
\text { complications }\end{array}$ & $\begin{array}{c}\text { Fisher's exact } \\
\text { test } p\end{array}$ \\
\hline Age [years]: & & & & 0.276 \\
\hline$\leq 60$ & $63(48.8 \%)$ & $25(43.9 \%)$ & $18(62.1 \%)$ & \\
\hline$>60$ & $66(51.2 \%)$ & $32(56.1 \%)$ & $11(37.9 \%)$ & \\
\hline Tumor type: & & & & 0.242 \\
\hline Adenocarcinoma & $84(65.1 \%)$ & $29(50.9 \%)$ & $16(55.2 \%)$ & \\
\hline Squamous cell & $41(31.8 \%)$ & $27(47.4 \%)$ & $13(44.8 \%)$ & \\
\hline Other histology & $4(3.1 \%)$ & $1(1.8 \%)$ & $0(0 \%)$ & \\
\hline Neoadjuvant therapy: & & & & 0.469 \\
\hline Yes & $107(82.9 \%)$ & $43(75.4 \%)$ & $24(82.8 \%)$ & \\
\hline No & $22(17.1 \%)$ & $14(24.6 \%)$ & $5(17.2 \%)$ & \\
\hline ASA score: & & & & 0.0002 \\
\hline I & $5(3.9 \%)$ & $2(3.5 \%)$ & $0(0 \%)$ & \\
\hline$\|$ & $72(55.8 \%)$ & $31(54.4 \%)$ & $4(13.8 \%)$ & \\
\hline III & $52(40.3 \%)$ & $24(42.1 \%)$ & $25(86.2 \%)$ & \\
\hline Procedure type: & & & & 0.302 \\
\hline MIE laparoscopic & $106(82.2 \%)$ & 49 (86.0\%) & $21(72.4 \%)$ & \\
\hline MIE thoracoscopic & $23(17.8 \%)$ & $8(14.0 \%)$ & $8(27.6 \%)$ & \\
\hline Anastomotic fistula: & & & & 0.124 \\
\hline Yes & $120(93.0 \%)$ & 49 (86.0\%) & $24(82.8 \%)$ & \\
\hline No & $9(7.0 \%)$ & $8(14.0 \%)$ & $5(17.2 \%)$ & \\
\hline TNM stage: & & & & 0.090 \\
\hline Complete pathological response & $22(17.1 \%)$ & $12(21.1 \%)$ & $4(13.8 \%)$ & \\
\hline I.A & $12(9.3 \%)$ & $8(14.0 \%)$ & $2(6.9 \%)$ & \\
\hline I.B & $12(9.3 \%)$ & $12(21.1 \%)$ & $1(3.4 \%)$ & \\
\hline II.A & $27(20.9 \%)$ & $5(8.8 \%)$ & $5(17.2 \%)$ & \\
\hline II.B & $15(11.6 \%)$ & $8(14 \%)$ & $4(13.8 \%)$ & \\
\hline III.A & $16(12.4 \%)$ & $4(7.0 \%)$ & $3(10.3 \%)$ & \\
\hline III.B & $10(7.8 \%)$ & $2(3.5 \%)$ & $5(17.2 \%)$ & \\
\hline III.C & $12(9.3 \%)$ & $5(8.8 \%)$ & $3(10.3 \%)$ & \\
\hline Clavien-Dindo: & & & & $<0.0001$ \\
\hline 1 & $26(27.7 \%)$ & $7(13.7 \%)$ & $0(0 \%)$ & \\
\hline 2 & $47(50.0 \%)$ & $29(56.9 \%)$ & $0(0 \%)$ & \\
\hline 3 & $13(13.8 \%)$ & $14(27.8 \%)$ & $4(13.8 \%)$ & \\
\hline 4 & $6(6.4 \%)$ & $1(2.0 \%)$ & $11(37.9 \%)$ & \\
\hline 5 & $2(2.1 \%)$ & $0(0 \%)$ & $14(48.3 \%)$ & \\
\hline
\end{tabular}


Table IV. Association of incidence of respiratory complications and quantitative parameters (Kruskal-Wallis test)

\begin{tabular}{|c|c|c|c|c|}
\hline Parameter & $\begin{array}{l}\text { Without respiratory } \\
\text { complications }\end{array}$ & $\begin{array}{l}\text { Mild respiratory } \\
\text { complications }\end{array}$ & $\begin{array}{l}\text { Severe respiratory } \\
\text { complications }\end{array}$ & Kruskal-Wallis $p$ \\
\hline Age [years]: & & & & 0.644 \\
\hline Median & 61.0 & 63.0 & 59.0 & \\
\hline Minimum & 34 & 44 & 37 & \\
\hline Maximum & 78 & 79 & 81 & \\
\hline Lymphadenectomy: & & & & 0.366 \\
\hline Median & 11.0 & 10.0 & 11.0 & \\
\hline Minimum & 2 & 0 & 3 & \\
\hline Maximum & 27 & 38 & 21 & \\
\hline Blood loss [ml]: & & & & 0.713 \\
\hline Median & 450 & 410 & 400 & \\
\hline Minimum & 100 & 120 & 140 & \\
\hline Maximum & 3000 & 3500 & 3500 & \\
\hline Hospital stay [day]: & & & & $<0.0001$ \\
\hline Median & 15.0 & 18.0 & 28.0 & \\
\hline Minimum & 9 & 11 & 9 & \\
\hline Maximum & 119 & 73 & 76 & \\
\hline
\end{tabular}

death was reported in $54 \%$, leading to secondary respiratory failure in $81 \%$ [7]. Siewert et al. published a cohort of 432 stage I-IV patients (according to the UICC classification), who underwent transthoracic or transhiatal esophageal resection. Respiratory complications (pneumonia or ARDS) developed in $22.9 \%$. Baba et al. reported RC in $23.6 \%$ after three-space esophageal dissection [12]. The occurrence of RC is particularly increased in elderly patients, in chronic nicotine abuse, malnutrition, pulmonary dysfunction and immune deficiency $[7,11]$. Further factors related to the development of RC include the operating times and estimated blood loss, $\mathrm{FEV}_{1}$ reduction on spirometry, neoadjuvant therapy, and the development of anastomotic leakage [13-16]. The incidence of $\mathrm{RC}$ can be influenced by the preoperative improvement of lung function and gentle anesthesia with early extubation $[17,18]$. Our experience supports the possible reduction of RC following preemptive antibiotic therapy with a significant decrease in mortality. This approach requires further studies.

If there is no general or local contraindication present, we prefer the minimally invasive approach for the resection phase of the procedure, anticipating the prevention of RC. Early weaning within $24 \mathrm{~h}$ after surgery and general and respiratory rehabilitation are required according to the postoperative recovery recommendations.

To unify the reporting of the post-esophagectomy complications, the "Consensus on Standardization of Data Collection for Complications Associated with Esophagectomy: Esophagectomy Complications Consensus Group (ECCG)" was published in 2015 [19]. According to this consensus, RC were divided into pneumonia, pleural effusions requiring thoracic drainage, pneumothorax requiring therapy, atelectasis requiring bronchoscopic treatment, acute aspiration, tracheobronchial injury, chest drainage for more than 10 days due to air leakage, respiratory failure requiring reintubation, and ARDS [20]. In particular, respiratory failure with reintubation and ARDS contribute to the increased mortality, while the other less serious complications are reflected in the increased postoperative morbidity. One of the most serious RC is the shock lung (ARDS), which was first described by Ausbagh et al. in 1967 in patients with an oxygen- 
ation disorder, reduced lung compliance and bilateral pulmonary infiltrates. The syndrome is exhibited in severe acute diseases with the clinical manifestation within 12-48 h. The prognosis is serious with the reported mortality of 30-60\% [21]. Zhang et al. demonstrated a significantly higher incidence of pneumonia in patients after chemoradiation in a study evaluating the effect of neoadjuvant chemotherapy and chemoradiation and the incidence of RC. There was no difference in the occurrence of ARDS, atelectasis, pleural effusions and respiratory failure in these two groups [22]. The literature does not describe a significant difference in the occurrence of RC in esophageal resection performed by the transthoracic or transhiatal approach [14]. Wan et al. did not observe a significant decrease in the incidence of RC following the thoracolaparoscopic method with cervical anastomosis compared to a control group of patients managed by classical McKeown esophagectomy [22]. We demonstrated significantly higher occurrence of $\mathrm{RC}$ in higher ASA scores and in Clavien-Dindo classes $\mathrm{IV}-\mathrm{V}$, underlining the negative impact of RC on mortality. We did not observe significantly higher incidence of RC in elderly patients in the age group over 60 years and in patients following neoadjuvant therapy. The reported results also show insignificant differences in the development of RC in patients managed via the minimally invasive transhiatal or thoracoscopic approach. The leak was not a cause of more frequent RC according to our statistical analysis [23]. The incidence of $R C$ is generally considered to be a negative prognostic factor determining longterm outcomes after esophagectomy [24].

\section{Conclusions}

It is not possible to eliminate post-esophagectomy RC in esophageal cancer patients completely. The results of our center show a higher occurrence of $\mathrm{RC}$ in polymorbid patients and patients with severe complications according to the Clavien-Dindo classification. As for the other parameters considered, there was no significant difference in the development of RC. The preoperative microbiological examination with subsequent targeted antibiotic prophylaxis and preemptive therapy of RC and the impact on morbidity require further studies.

\section{Conflict of interest}

The authors declare no conflict of interest.

\section{References}

1. Wormuth JK, Heitmiler RF. Esophageal conduit necrosis. Thorac Surg Clin 2006; 16: 11-22.

2. Dindo D, Demartines N, Clavien PA. Classification of surgical complications: a new proposal with evaluation in a cohort of 6336 patients and results of a survey. Ann Surg 2004; 240: 205-13.

3. Meng F, Li Y, Ma H, et al. Comparison of outcomes of open and minimally invasive esophagectomy in 183 patients with cancer. J Thorac Dis 2014; 6: 1218-24.

4. Zhai C, Liu Y, Li W, et al. A comparison of short-term outcomes between Ivor-Lewis and McKeown minimally invasive esophagectomy. J Thorac Dis 2015; 7: 2352-8.

5. Biere SS, van Berge Henegouwen MI, Maas KW, et al. Minimally invasive versus open oesophagectomy for patients with oesophageal cancer: a multicentre, open-label, randomized controlled trial. Lancet 2012; 379: 1887-92.

6. Ott K, Bader FG, Lordick F, et al. Surgical factors influence the outcome after Ivor-Lewis esophagectomy with intrathoracic anastomosis for adenocarcinoma of the esophagogastric junction: a consecutive series of 240 patient at an experienced center. Ann Surg Oncol 2009; 16: 1017-25.

7. Atkins BZ, Shan AS, Kelley A, et al. Reducing hospital morbidity and mortality following esophagectomy. Ann Thorac Surg 2004; 78: 1170-6.

8. Wlodarczyk J, Kużdżał J. Stenting as a palliative method in the management of advanced squamous cell carcinoma of the oesophagus and gastro-oesophageal junction. Videosurgery Miniinv 2016; 11: 1-8.

9. Zhang Z, Zhang H. Impact of neoadjuvant chemotherapy and chemoradiotherapy on postoperative cardiopulmonary complications in patiens with esophageal cancer. Dis Esophagus 2017; 30: 1-7.

10. Dumont P, Wihlm JM, Hentz JG, et al. Respiratory complications after surgical treatment of esophageal cancer. A study of 309 patients according to the type of resection. Eur J Cardiothorac Surg 1995; 9: 539-43.

11. Ferguson MK, Durkin AE. Preoperative prediction of the risk of pulmonary complications after esophagectomy for cancer. J Thorac Cardiovasc Surg 2002; 123: 661-9.

12. Baba M, Aikou T, Yoshinaka H, et al. Low-term results of subtotal esophagectomy with three-field lymphadenectomy for carcinoma of the thoracic esophagus. Ann Surg 1994; 219: 108-13.

13. Dahn D, Martell J, Vorwerk H, et al. Influence of irradiated lung volumes on perioperative morbidity and mortality in patients after neoadjuvant radiochemotherapy for esophageal cancer. Int J Radiat Oncol Biol Phys 2010; 77: 44-52.

14. Xing XZ, Gao Y, Wang HJ, et al. Assessment of a predictive score for pulmonary complications in cancer patients after esophagectomy. World J Emerg Med 2016; 7: 44-9.

15. Tandon S, Batchelor A, Bullock R, et al. Perioperative risk factor for acute lung injury after elective esophagectomy. Br J Anesth 2001; 86: 633-8.

16. Bartels H, Stein HJ, Siewert JR. Risk analysis in esophageal surgery. Recent Result Cancer Res 2000; 155: 89-96.

17. Dumont P, Wihlm JM, Hentz JG, et al. Respiratory complications after surgical treatment of esophageal cancer A study of 309 
patients according to the type of resection. Eur J Cardiothorac Surg 1995; 9: 539-43.

18. Horáková M, Lubušká L, Kolář K, et al. Individualized prophylaxis in patients with esophageal replacement for cancer. Surg Infect 2015; 16: 513-7.

19. Low DE, Alderson D, Cecconello I, et al. International consensus on standardization of data collection for complications associated with esophagectomy: esophagectomy complications consensus group (ECCG). Ann Surg 2015; 262: 286-94.

20. Wiedermann HP, Wheeler AP, Bernard GR, et al. Comparison of two fluid-management strategies in acute lung injury. N Eng J Med 2006; 354: 256-75.

21. Łochowski MP, Kozak J. Video-assisted thoracic surgery complications. Videosurgery Miniinv 2014; 9: 495-500.

22. Wan J, Che Y, Kang N, Zhang R. Surgical method, postoperative complications, and gastrointestinal motility of thoraco-laparoscopy 3-field esophagectomy in treatment of esophageal cancer. Med Sci Monit 2016; 22: 2056-65.

23. Baba Y, Yoshida N, Shigaki H, et al. Prognostic impact of postoperative complications in 502 patients with surgically resected esophageal squamous cell carcinoma: a retrospective single-institution study. Ann Surg 2016; 264: 305-11.

24. Lerut T, Moons J, Coosemans W, et al. Postoperative complications after transthoracic esophagectomy for cancer of the esophagus and gastroesophageal junction are correlated with early cancer recurrence: role of systematic grading of complications using the modified Clavien classification. Ann Surg 2009; 25: 798-807.

Received: 11.02.2018, accepted: 16.06.2018. 